\title{
NineML - a description language for spiking neuron network modeling: the abstraction layer
}

\author{
Ivan Raikov ${ }^{1,3^{*}}$, INCF Multiscale Modeling Taskforce ${ }^{2}$ \\ From Nineteenth Annual Computational Neuroscience Meeting: CNS*2010 \\ San Antonio, TX, USA. 24-30 July 2010
}

With an increasing number of studies related to largescale neuronal network modeling, the International Neuroinformatics Coordinating Facility (INCF) has identified a need for standards and guidelines to ease model sharing and facilitate the replication of results across different simulators. To create such standards, the INCF has formed a program on Multiscale Modeling to develop a common standardized description language for neuronal network models. The name of the proposed standard is Network Interchange for Neuroscience Modeling Language (NineML) and its first version is aimed at descriptions of large networks of spiking neurons. The design of NineML is divided in two semantic layers: an abstraction layer that provides the core concepts, mathematics and syntax with which model variables and state update rules are explicitly described and a user layer that provides a syntax to specify the instantiation and parameterization of a network model in biological terms.

The key concepts of spiking neuron network modeling are 1) spiking neurons 2) synapses 3) populations of neurons and 4) connectivity patterns across populations of neurons. Accordingly, the INCF task force on multiscale modeling has identified a set of mathematical abstractions that are capable of representing these concepts in a computer language. First, we propose a flexible block diagram notation for describing spiking dynamics. The notation represents continuous and discrete variables, their evolution according to a set of rules such as a system of ordinary differential equations, and the conditions that induce a regime change, such as the transition from subthreshold mode to spiking and refractory modes. The notation we have developed is an

\footnotetext{
* Correspondence: raikov@oist.jp

'Computational Neuroscience Unit, Okinawa Institute of Science and Technology, Okinawa, Japan
}

explicit formalization of event handling and is an important step in ensuring model simulation consistency.

In addition, the abstraction layer provides the notation to describe a variety of topographical arrangements of neurons and populations, and to describe random connectivity patterns between neuronal populations, based on structural properties of neuronal networks.

\section{Acknowledgments}

This work is supported by the International Neuroinformatics Coordinating Facility (INCF). Members of this Task Force include primary contributors to projects including the Blue Brain Project, GENESIS-3, KInNeSS, MOOSE, NEURON, NEST, PYNN and NeuroML.

\section{Author details}

${ }^{1}$ Computational Neuroscience Unit, Okinawa Institute of Science and Technology, Okinawa, Japan. ${ }^{2}$ INCF Secretariat, Karolinska Institutet, Nobels väg 15 A, SE-171 77 Stockholm, Sweden. ${ }^{3}$ University of Antwerp, Antwerp, Belgium.

Published: 20 July 2010

doi:10.1186/1471-2202-11-S1-P66

Cite this article as: Raikov and : NineML - a description language for spiking neuron network modeling: the abstraction layer. BMC

Neuroscience 2010 11(Suppl 1):P66.

Submit your next manuscript to BioMed Central and take full advantage of:

- Convenient online submission

- Thorough peer review

- No space constraints or color figure charges

- Immediate publication on acceptance

- Inclusion in PubIMed, CAS, Scopus and Google Scholar

- Research which is freely available for redistribution

Submit your manuscript at www.biomedcentral.com/submit 